\title{
An efficient quorum-based rendezvous scheme for multi-radio cognitive radio networks
}

\begin{abstract}
Rendezvous is an initial and vital process for establishing data communications between devices in cognitive radio networks. Channel hopping $(\mathrm{CH})$ provides an effective method for achieving rendezvous without relying on a dedicated common control channel. Most of the existing rendezvous schemes are designed for single-radio devices. Due to the dropping cost of wireless transceivers, the use of multiple radios to significantly reduce the rendezvous delay becomes economically feasible. In this paper, we propose a deterministic multi-radio rendezvous scheme that exploits the combinatorial features of grid quorum systems. We refer to our proposed scheme as multi-grid-quorum channel hopping (MGQ-CH). Our scheme uses multiple overlapped grid quorums to map the available channels at each device to its radios. We derive the theoretical upper-bound of the maximum time-to-rendezvous of MGQ- $\mathrm{CH}$. Furthermore, we conduct simulations to study the performance of MGQ-CH under various system parameters and compare it with the state-of-the-art multi-radio rendezvous algorithms. The simulation results demonstrate the superior performance of MGQ-CH compared to previous schemes.
\end{abstract}

